\title{
Potencial de óleos essenciais de plantas medicinais no controle de fitopatógenos
}

FONSECA, M.C.M..1; LEHNER, M.S.2; GONÇALVES, M.G. ;; PAULA JÚNIOR, T.J.'; SILVA, A.F.'; BONFIM, F.P.G.4; PRADO, A.L. ${ }^{3}$

'EPAMIG-UREZM, Vila Gianetti, 46. Campus da UFV. CEP 36570-000, Viçosa-Brasil; 2Universidade Federal de Viçosa; ${ }^{3}$ Graduandas Agronomia - UFV/EPAMIG; ${ }^{4}$ UNESP - FCA, Rua José Barbosa de Barros, $n^{\circ} 1780$, CEP: 18610-307, Botucatu-Brasil.maira@epamig.br

RESUMO: Além do valor como recurso terapêutico, plantas medicinais também possuem potencial para serem utilizadas como fonte de princípios ativos contra fitopatógenos. O objetivo deste trabalho foi avaliar o efeito de óleos essenciais das espécies medicinais Baccharis dracunculifolia (alecrim-do-campo), Schinus terebinthifolius (aroeirinha) e Porophyllum ruderale (arnica-brasileira) sobre o crescimento dos fungos fitopatogênicos Fusarium oxysporum f. sp. phaseoli (Fop), F. solani f. sp. phaseoli (Fsp), Sclerotinia sclerotiorum (Ss), S. minor (Sm), Rhizoctonia solani (Rs), Sclerotium rolfsii (Sr) e Macrophomina phaseolina (Mp). Avaliou-se em placas de Petri o crescimento radial desses fungos em meio batata-dextrose-ágar (BDA) com cinco concentrações $\left(0,250,500,1000\right.$ e $\left.3000 \mathrm{mg} \mathrm{L}^{-1}\right)$ dos óleos essenciais. Discos de micélio ( $5 \mathrm{~mm}$ de diâmetro) de cada fungo em crescimento foram transferidos para placas de Petri que foram mantidas a $23^{\circ} \mathrm{C}$ no escuro por 48 horas. O óleo essencial de alecrim-do-campo foi o mais eficiente na redução do crescimento micelial de todos os fungos, com inibição completa quando se utilizou a concentração de $3000 \mathrm{mg} \mathrm{L}^{-1}$. A redução de crescimento variou de $29 \%$ (Fs) a $80 \%$ (Rs) a $250 \mathrm{mg} \mathrm{L}^{-1}$ do óleo essencial de alecrim-do-campo; a $500 \mathrm{mg} \mathrm{L}^{-1}$, variou de $29 \%(\mathrm{Fs})$ a $98 \%(\mathrm{Sr})$; e a $1000 \mathrm{mg} \mathrm{L}^{-1}$, de $41 \%(\mathrm{Fs})$ a $100 \%(\mathrm{Sr})$. A redução do crescimento dos fungos pelo óleo de aroeirinha na concentração de $3000 \mathrm{mg} \mathrm{L}^{-1}$ variou de $27 \%$ (Fsp) a $74 \%$ (Rs). Nessa concentração, o óleo de arnica-brasileira reduziu o crecimento micelial de Ss em $72 \%$, o de Rs em $80 \%$ e o de Mp em $82 \%$, sem efeitos significativos sobre o crescimento micelial de Fsp e Fop. Conclui-se que os óleos essenciais de alecrim-do-campo, aroeirinha e arnica-brasileira possuem potencial para o controle dos fungos fitopatogênicos estudados, com destaque para o óleo de alecrim-do-campo.

Palavras-chave: controle alternativo, alecrim-do-campo, aroeirinha, arnica-brasileira.

ABSTRACT: Potential of essential oils from medicinal plants to control plant pathogens. In addition to their value as therapeutic resources, medicinal plants also have the potential to be used as a source of alternative compounds against plant pathogens. The objective of this study was to evaluate the effect of essential oils extracted from the medicinal species Baccharis dracunculifolia, Schinus terebinthifolius and Porophyllum ruderale on the growth of the fungal plant pathogens Fusarium oxysporum f. sp. phaseoli (Fop), F. solani f. sp. phaseoli (Fsp), Sclerotinia sclerotiorum (Ss), S. minor (Sm), Rhizoctonia solani (Rs), Sclerotium rolfsii $(\mathrm{Sr})$ and Macrophomina phaseolina (Mp). The radial mycelial growth of the fungi was evaluated on potato dextrose agar (PDA) in Petri dishes with five concentrations $(0,250,500,1000$ and $3000 \mu \mathrm{L} \mathrm{L-1}$ ) of the essential oils. Mycelial discs (5 mm diameter) of the growing colonies of each fungus were transferred to Petri dishes, which were maintained at $23^{\circ} \mathrm{C}$ in the dark for 48 hours. The essential oil of $B$. dracunculifolia was the most effective oil in the reduction of the mycelial growth of all fungi. It completely inhibited their growth at $3000 \mathrm{mg} \mathrm{L}^{-1}$. At $250 \mathrm{mg} \mathrm{L}^{-1}$, the growth reduction caused by the oil of $B$. dracunculifolia varied from $29 \%$ (Fs) to $80 \%$ (Rs); at $500 \mathrm{mg} \mathrm{L}^{-1}$, it varied from $29 \%(\mathrm{Fs})$ to $98 \%(\mathrm{Sr})$; and at $1000 \mathrm{mg} \mathrm{L}^{-1}$, it varied from $41 \%$ (Fs) to $100 \%(\mathrm{Sr})$. The reduction of the mycelial growth caused by the oil of $S$. terebinthifolius at 3000 $\mathrm{mg} \mathrm{L}^{-1}$ varied from $27 \%$ (Fsp) to $74 \%$ (Rs). At this concentration, the oil of $P$. ruderale reduced the mycelial growth of Ss by $72 \%$, of Rs by $80 \%$ and of Mp by $82 \%$, without significant effects on the mycelial growth of Fsp and Fop. We conclude that the essential oils of $B$. dracunculifolia, $S$. terebinthifolius and $P$. ruderale have the potential to be used to control the plant pathogens tested, especially the oil of $B$. dracunculifolia.

Kew words: alternative control, Baccharis dracunculifolia, Schinus terebinthifolius, Porophyllum ruderale. 


\section{INTRODUÇÃO}

O Brasil é um dos maiores consumidores de defensivos agrícolas do mundo. São gastos, anualmente, cerca de 2,5 bilhões de dólares com a aquisição desses produtos, sendo o país responsável pelo consumo de cerca de $50 \%$ da quantidade de defensivos utilizados na América Latina (MMA, 2000). Como consequência, ocorrem graves desequilíbrios ambientais, resultando na contaminação de alimentos, animais e reservas hídricas, e ocasionando a redução na qualidade e na expectativa de vida da população. Desse modo se faz necessária a busca de medidas alternativas de manejo fitossanitário compatíveis com a qualidade ambiental visada no manejo sustentável. O controle de doenças, na maioria das vezes, tem sido feito com a utilização de fungicidas sintéticos. Apesar da facilidade de aquisição e de uso, problemas como o desenvolvimento de resistência de patógenos devido ao uso contínuo e à alta toxicidade de determinados produtos, estão frequentemente associados à utilização exclusiva do controle químico (Fragoso et al., 2002). Soma-se a esses fatores o custo elevado dos produtos que onera a produção, muitas vezes realizada por produtores familiares. Além disso, nos sistemas de produção em que a utilização do controle químico não é permitida, como por exemplo no cultivo orgânico, há necessidade de métodos alternativos com eficiência comprovada para o controle de pragas e doenças (Venzon et al., 2006). Considerando-se o valor das plantas medicinais não apenas como recurso terapêutico, mas também como fonte de controle alternativo de pragas e doenças, torna-se importante estabelecer linhas de ações voltadas ao desenvolvimento de técnicas de manejo sustentável, utilizando espécies que possibilitem contribuir para a manutenção do equilíbrio dos ecossistemas tropicais (Reis, 1996; Sheldon et al., 1997).

Pesquisas visando ao controle alternativo de pragas e doenças, principalmente daquelas que provocam danos econômicos à agricultura, através do emprego de óleos essenciais e extratos vegetais (Schwan-Estrada \& Stangarlin, 2005) têm aumentado consideravelmente nos últimos anos e revelado seu potencial. Algumas plantas apresentam diversas substâncias em sua composição química, muitas delas com potencial fungicida ou fungistático, as quais devem ser estudadas para utilização direta do produtor rural, bem como para servir de matéria prima para formulação de novos produtos (Garcia et al, 2012). Dentre estas plantas encontram-se Baccharis dracunculifolia DC. (alecrim-do-campo), Porophyllum ruderale (Jacq.) Cass. (arnica-brasileira) e Schinus terebinthifolius Raddi. (aroeirinha) (Devincenzi et al., 1996; Fenner et al., 2006; Souza et al., 2008). Pouco se sabe sobre o efeito de óleos essencias dessas espécies medicinais sobre alguns fungos fitopatogênicos que atacam culturas economicamente importantes. Tais informações poderiam contribuir para o controle alternativo de algumas doenças e desenvolvimento futuro de novos produtos.

O objetivo deste trabalho foi avaliar in vitro o efeito de óleos essenciais de $B$. dracunculifolia, $P$. ruderale e $S$. terebinthifolius sobre o crescimento dos fungos fitopatogênicos Fusarium oxysporum Schlecht. f. sp. phaseoli Kendrick \& Snyder (Fop), Fusarium solani f. sp. phaseoli W.C. Snyder \& H.N. Hansen (Fsp), Sclerotinia sclerotiorum (Lib.) De Bary (Ss), Sclerotinia minor Jagger (Sm), Rhizoctonia solani Kühn (Rs), Sclerotium rolfsii Sacc. (Sr) e Macrophomina phaseolina (Tassi) Goid. (Mp).

\section{MATERIAL E MÉTODOS}

O experimento foi conduzido no Laboratório de Fitopatologia da Empresa de Pesquisa Agropecuária de Minas Gerais (EPAMIG), situado em Viçosa-MG.

\section{Obtenção dos isolados}

Os fungos Fop, Fsp, Mp e Ss foram coletados em feijão-comum (Phaseolus vulgaris L.); Sr, em batata (Solanum tuberosum L.); e Rs, em feijão-caupi (Vigna unguiculata (L.) Walp.). Dos fungos Mp, Ss e Sr coletaram-se escleródios; de Rs, Fop e Fsp, seções de raízes infectadas.

Escleródios ou seções de raízes foram desinfestados em solução de etanol $70 \%$ por $1 \mathrm{~min}$, seguido de hipoclorito de sódio $1 \%$ por 3 min. Depois, escleródios e seções de raízes foram lavados em água esterilizada e transferidos para placas de Petri de $9 \mathrm{~cm}$ de diâmetro contendo $15 \mathrm{~mL}$ de meio batata-dextrose-ágar (BDA) com cloranfenicol (100 $\left.\mathrm{mg} \mathrm{L}^{-1}\right)$. As placas foram mantidas no escuro por 10 dias, a $23^{\circ} \mathrm{C} \pm 2{ }^{\circ} \mathrm{C} \mathrm{e}$, em seguida, armazenadas a $4^{\circ} \mathrm{C}$. A confirmação da espécie de cada isolado foi feita com base em características da colônia em meio BDA. No caso de Fop e Fsp, além desse critério, procedeu-se também à inoculação dos fungos em plantas de feijão. Destas plantas, o fungo foi reisolado e, com auxílio de microscópio, confirmou-se a presença de macro e microconídios do fungo pertenente ao gênero Fusarium sp.

\section{Efeito de óleos essenciais sobre o crescimento micelial dos fitopatógenos}

Os óleos essenciais foram obtidos atraves da hidrodestilação em aparelho Clevenger, a partir da parte aérea das espécies medicinais selecionadas, dissolvidos em dimetilsulfóxido (DMSO) e incorporados ao meio BDA nas concentrações 
de $0,250,500,1000$ e $3000 \mathrm{mg} \mathrm{L}^{-1}$. A mistura dos óleos em BDA foi realizada quando o meio estava em fase líquida, em temperatura próxima de $45^{\circ} \mathrm{C}$. Placas com BDA e DMSO foram utilizadas como controle (dose zero). Para obter crescimento ativo dos fungos, Mp, Rs, Sr e Ss foram repicados três dias antes do início do experimento, e Fop e Fsp sete dias antes. Discos de micélio de $5 \mathrm{~mm}$ de diâmetro de cada fungo foram transferidos para o centro de placas de Petri contendo BDA acrescido dos óleos essenciais nas cinco concentrações. As placas foram mantidas a $23^{\circ} \mathrm{C}$ no escuro, ultilizando-se o delineamento inteiramente casualizado, com quatro repetições.

O diâmetro das colônias (Dc) foi obtido da média de duas medidas diametralmente opostas, com o uso de paquímetro digital. A primeira medição foi realizada com $24 \mathrm{~h}$ de incubação e a segunda com $48 \mathrm{~h}$, quando o micélio de Ss no tratamento controle cobriu toda a superfície do meio. A percentagem de redução do crescimento micelial dos fungos em função das concentrações de óleos essenciais foi calculada com o Dc mensurado com $48 \mathrm{~h}$ de acordo com a seguinte equação:

\author{
\% redução do Dc tratamento controle - Dc tratamento com óleo $\times 100$ \\ crescimento micelial \\ Dc tratamento controle
}

Foram realizadas análises de regressão, escolhendo-se o melhor modelo que apresentasse significância e sentido biológico para explicar o efeito das concentrações crescentes dos óleos essenciais sobre o crescimento micelial dos fungos. Os dados foram apresentandos em gráficos contendo a média e desvio padrão do diâmetro de colônia dos fungos em cada concentração dos óleos essenciais.

\section{RESULTADOS E DISCUSSÃO}

Testes in vitro são geralmente utilizados para a seleção inicial de produtos alternativos capazes de controlar fitopatógenos. No presente estudo, avaliou-se o efeito de óleos essenciais extraídos de três espécies medicinais sobre patógenos que estão amplamente distribuídos em todo o mundo, com centenas de espécies de plantas hospedeiras, sendo afetadas muitas culturas economicamente importantes (Boland e Hall, 1994; Goulart \& Fialho, 1998).

O óleo de aroeirinha (S. terebinthifolius) na concentração de $3000 \mathrm{mg} \mathrm{L}^{-1}$, promoveu a redução de crescimento dos fungos, a qual variou de $27 \%$ (Fsp) a $74 \%$ (Rs) em relação ao controle (Figura 1). Com esse óleo, o crescimento de Fsp foi pouco afetado e o de Fop foi acentuadamente reduzido apenas em concentração de $3000 \mathrm{mg} \mathrm{L}^{-1}$. O diâmetro de colônia de Fop no tratamento controle foi de 18 $\mathrm{mm}$ e na concentração de $3000 \mathrm{mg} \mathrm{L}^{-1}$ foi de 8,6 $\mathrm{mm}$. O diâmetro de colônia de Fsp nos respectivos tratamentos foram 12,8 $\mathrm{mm}$ e $9,4 \mathrm{~mm}$ (Figura 1). $\mathrm{Sm}$ e Sr tiveram reduções de crescimento de $60 \%$ e $72 \%$, resctivamente, em concentração de 3000 $\mathrm{mg} \mathrm{L}^{-1}$ do óleo de aroeirinha.

O óleo extraído de arnica-brasileira $(P$. ruderale) reduziu acentuadamente o crescimento micelial da maioria dos fungos a partir de 500 $\mathrm{mg} \mathrm{L}^{-1}$ (Figura 2). Os fungos do gênero Fusarium foram pouco afetados (Fsp) ou afetados apenas na concentração de $3000 \mathrm{mg} \mathrm{L}^{-1}$ (Fop). O óleo de arnica-brasileira na concentração de $3000 \mathrm{mg} \mathrm{L}^{-1}$ reduziu o crecimento micelial de Ss em $72 \%$, o de $\mathrm{Rs}$ em $80 \%$ e o de $\mathrm{Mp}$ em $82 \%$, em relação ao controle. Com 1000 mg L-1, esse óleo reduziu o crescimento de Ss, Rs e Mp em $26 \%, 40 \%$ e $48 \%$, respectivamente.

O óleo de alecrim-do-campo ( $B$. dracunculifolia) reduziu acentuadamente o crescimento de todos os fungos (Figura 3). Para três deles (Rs, $\mathrm{Sr}$ e Sm), a redução do crescimento foi de $100 \%$ quando utilizou-se a concentração de $3000 \mathrm{mg} \mathrm{L}^{-1}$. Vale destacar que o óleo de alecrim-docampo foi também muito eficiente em concentrações menores. Na concentração de $250 \mathrm{mg} \mathrm{L}^{-1}$, a redução de crescimento dos fungos variou de $29 \%$ (Fs) a $80 \%$ (Rs); a $500 \mathrm{mg} \mathrm{L}^{-1}$, variou de $29 \%$ (Fs) a $98 \%$ (Sr); e a $1000 \mathrm{mg} \mathrm{L}^{-1}$, a redução de crescimento variou de $41 \%$ (Fs) a $100 \%$ (Sr).

Em seus estudos, Salgado et al. (2003) observaram atividades fungitóxicas em $F$. oxysporum na concentração de $500 \mathrm{mg} \mathrm{L}^{-1}$ do óleo das três espécies de eucalipto testadas. Outros resultados no controle de fungos do gênero Fusarium foram demonstrados por Singh et al. (1993), que verificaram o efeito fungicida e fungistático do óleo de menta (Mentha sp.) sobre 23 espécies de fitopatógenos, registrando total inibição do crescimento micelial, a partir de $2.000 \mathrm{mg} \mathrm{mL}^{-1}$.

Em pesquisa realizada por Oliveira et al. (2006), o óleo de Copaifera dukey (copaíba) foi eficiente na inibição do crescimento micelial dos fungos S. rolfsii, $M$. phaseolina e $R$. solani na concentração de $250 \mu \mathrm{L}$ de óleo $100 \mathrm{~mL}^{-1}$ de BDA. Conclui-se que o óleo essencial de alecrim-

Rev. Bras. PI. Med., Campinas, v.17, n.1, p.45-50, 2015. 


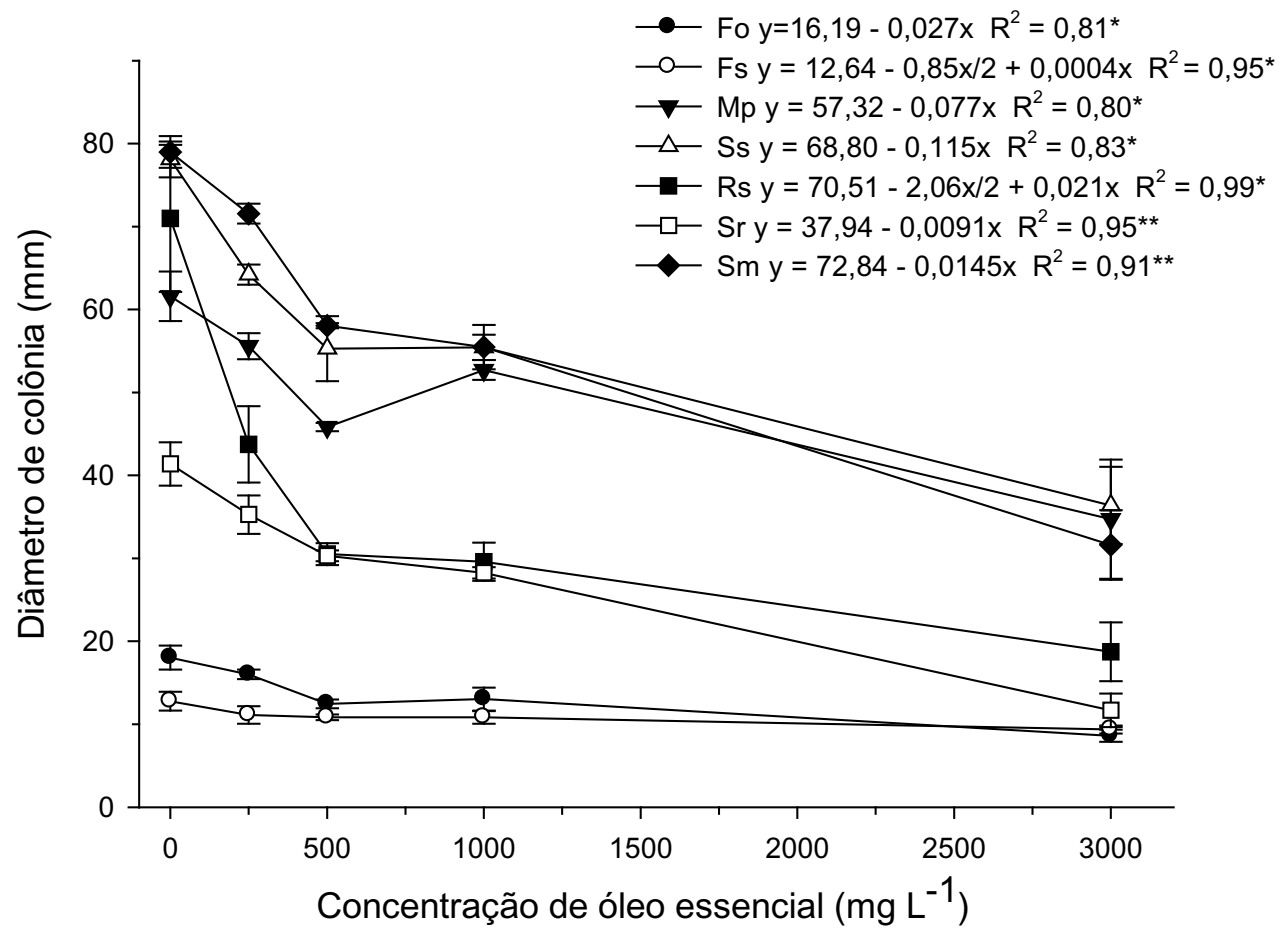

FIGURA 1. Efeito do óleo essencial de aroeirinha (Schinus terebinthifolius) incorporado ao meio BDA sobre o crscimento micelial de Fusarium oxysporum f.sp. phaseoli (Fop), Fusarium solani f.sp. phaseoli (Fsp), Macrophomina phaseolina (Mp), Sclerotinia sclerotiorum (Ss), Rhizoctonia solani (Rs), Sclerotium rolfsii (Sr) e Sclerotinia minor $(\mathrm{Sm})$. Médias \pm desvio padrão. * $P<0,01{ }^{* *} P<0,05$.

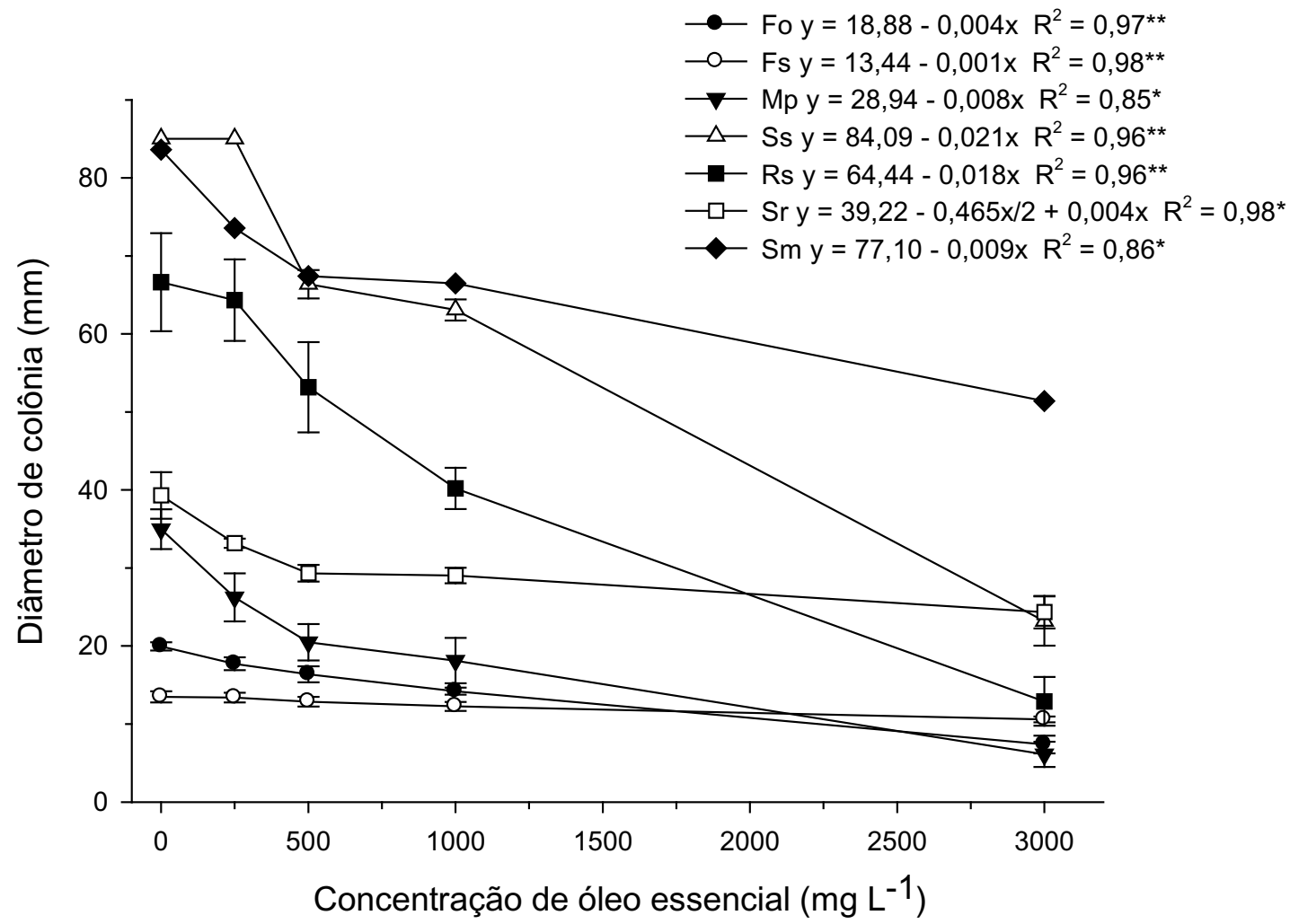

FIGURA 2. Efeito do óleo essencial de arnica-brasileira (Porophyllum ruderale) incorporado ao meio BDA sobre o crscimento micelial de Fusarium oxysporum f.sp. phaseoli (Fop), Fusarium solani f.sp. phaseoli (Fsp), Macrophomina phaseolina (Mp), Sclerotinia sclerotiorum (Ss), Rhizoctonia solani (Rs), Sclerotium rolfsii (Sr) e Sclerotinia minor (Sm). Médias \pm desvio padrão. ${ }^{*} P<0,01{ }^{* *} P<0,05$. 


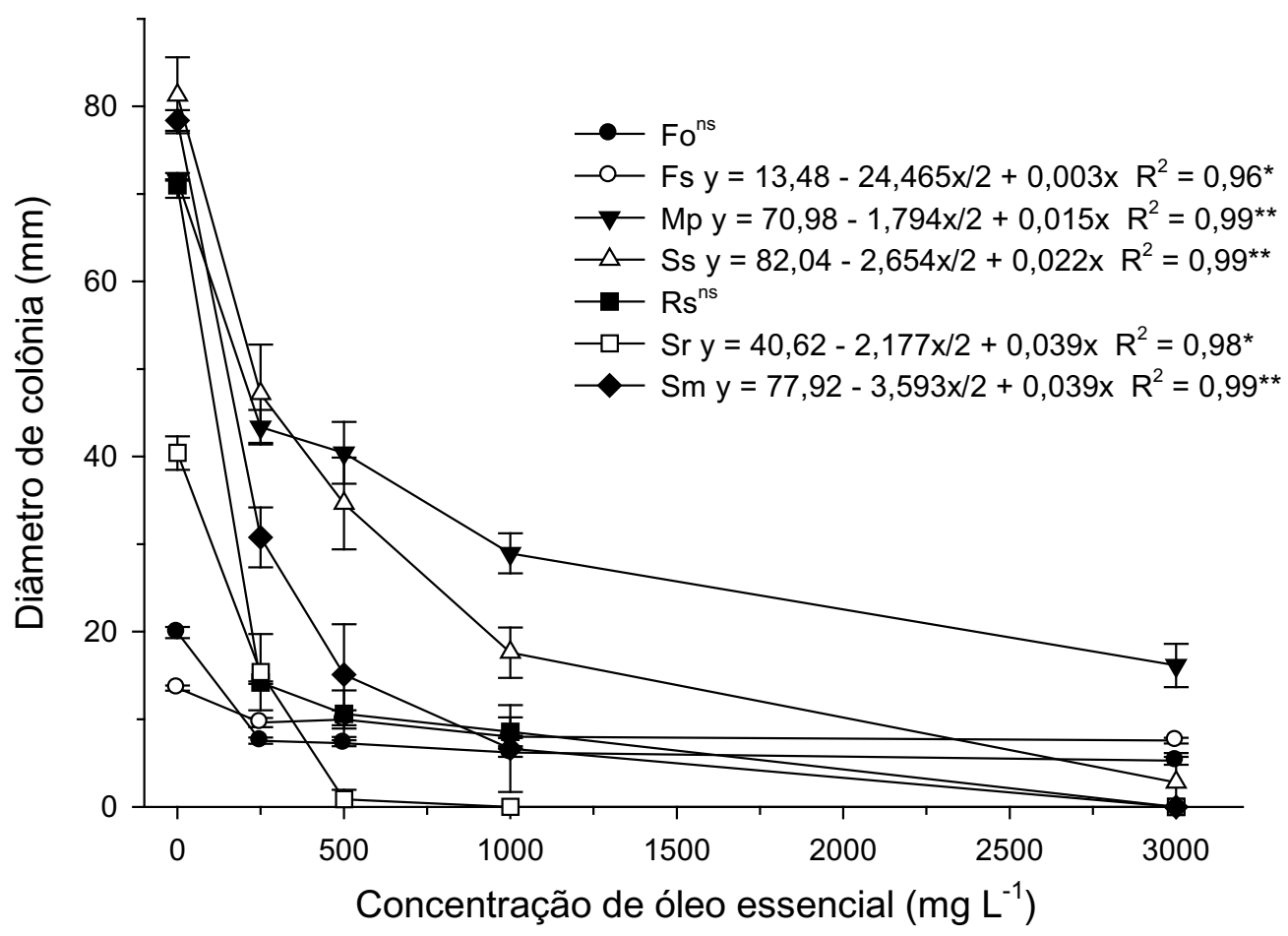

FIGURA 3. Efeito do óleo essencial de alecrim-do-campo (Baccharis dracunculifolia) incorporado ao meio BDA sobre o crscimento micelial de Fusarium oxysporum f.sp. phaseoli (Fop), Fusarium solani f.sp. phaseoli (Fsp), Macrophomina phaseolina (Mp), Sclerotinia sclerotiorum (Ss), Rhizoctonia solani (Rs), Sclerotium rolfsii (Sr) e Sclerotinia minor (Sm). Médias \pm desvio padrão. ${ }^{*} P<0,01^{* *} P<0,05$. ns não significativo pelo modelo linear ou de raiz quadrada.

do-campo (B. dracunculifolia) foi o mais eficiente na redução do crescimento micelial dos fungos em comparação com os óleos de aroeirinha ( $S$. terebinthifolius) e arnica-brasileira ( $P$. ruderale), sendo a redução do crescimento micelial diretamente proporcional ao aumento das concentrações, destacando-se como o mais promissor na inibição do crescimento micelial. O óleo essencial de alecrim-do-campo tem potencial para controle dos fitopatógenos testados, no entanto, apesar dos resultados promissores nos testes in vitro, estudos de campo são essenciais para confirmar o seu potencial de uso no controle destes fitopatógenos.

\section{AGRADECIMENTOS}

À FAPEMIG e ao CNPq pelo financiamento do projeto e pelas bolsas concedidas.

\section{REFERÊNCIAS}

BOLAND, G.J.; HALL, R. Index of plant hosts of Sclerotinia sclerotiorum. Canadian Journal of Plant Pathology, v.16, p. 93-108, 1994.

DEVINCENZI, I.A.A. Atividade fungitóxica e citotóxica do óleo essencial de Pororphyllum ruderale. In: SIMPÓSIO
DE PLANTAS MEDICINAIS DO BRASIL, 14,1996. Florianópolis. Anais... Florianópolis: UFSC 1996. 151 p.

FENNER, R.; BETTI, A.H.; MENTZ, L.A.; RATES, S.M. Plantas utilizadas na medicina popular brasileira com potencial atividade antifúngica. Revista Brasileira de Ciências Farmacêuticas, v. 42, p. 369-394, 2006.

FRAGOSO, D.B., GUEDES, R.N.C., PICANÇO, M.C., ZAMBOLIM, L. Inseticide use and organophosphate resistance in the coffee leaf miner Leucoptera coffeella (Lepidoptera: Lyonetiidae). Bulletin of Entomological Research, v. 92, p. 203-212, 2002.

GARCIA, R.A.; JULIATTI, F.C.; BARBOSA, K.A.G.; CASSEMIRO, T.A. Atividade antifúngica de óleos e extratos vegetais sobre Sclerotinia sclerotiorum. Bioscience Journal, v. 28, p. 48-57, 2012.

GOULART, A.C.P; FIALHO, W.F.B. Ocorrência de fungos em sementes de milho "Br 201" produzidas na região de Dourados, MS. Fitopatologia Brasileira, v. 23, p.79, 1998.

MMA (Ministério do Meio Ambiente). Informativo MMA, 2000. Disponível em: http:/

/www.mma.gov.br/port/ascom/imprensa/marco2000/ informma15.html.

OLIVEIRA, E.C.P.; LAMEIRA, O.A.; BARROS, P.L.C.; POLTRONIERI, L.B. 2006. Avaliação do óleo de copaíba (Copaifera) na inibição do crescimento micelial in vitro de fitopatógenos. Revista Ciências Agrárias, v. 46, p. 53-61, 2006.

REIS, M.S. Manejo sustentado de plantas medicinais em ecossistemas tropicais. In: DI STASI, L. C. (Org.).

Rev. Bras. PI. Med., Campinas, v.17, n.1, p.45-50, 2015. 
Plantas Medicinais: arte e ciência. Um guia de estudo multidisciplinar. São Paulo: Unesp. 1996. p. 199-215.

SALGADO, A.P.S.P.; CARDOSO, M.G.; SOUZA, P.E.; SOUZA, J.A.; ABREU, C.M.P.; PINTO, J.E.B.P. Avaliação da atividade fungitóxica de óleos essenciais de folhas de Eucalyptus sobre Fusarium oxysporum, Botrytis cinerea e Bipolares sorokiniana. Ciência e Agrotecnologia, v. 27, p. 249-254, 2003.

SCHWAN-ESTRADA, K.R.F., STANGARLIN, JR. Extratos e óleos essenciais de plantas medicinais na indução de resistência. In: CAVALCANTI, L.S.; DI PIERO, R.M.; CIA, P. et al. (Eds.). Indução de resistência em plantas a patógenos e insetos. Piracicaba: FEALQ. p. 125-132, 2005.

SHELDON, JW.; BALICK, MJ.; LAIRD, SA. Medicinal plants: can utilization and conservation coexist?
New York: The New York Botanical Garden. 1997. $104 p$.

SINGH, H.N.P.; PRASAD, M.M.; SINHA, K.K. Efficacy of leaf extracts of some medicinal plants against disease development in banana. Letters in Applied Microbiology, v. 17, p. 269-271, 1993.

SOUZA, A.P.; MARQUES, M.R.; MAHMOUD, T.S. et al. Bioprospecting inseticidal compounds from plants native to Mato Grosso do Sul, Brasil. Acta Botanica Brasilica, v. 22, p.1136-1140, 2008.

VENZON, M.; TUELHER, E.S.; BONOMO, I.S., TINOCO, R.S.; FONSECA, M.C.M.; PALLINI, A. Potencial de defensivos alternativos para o controle de pragas de cafeeiro. In: PAULA JUNIOR, T. J.; VENZON, M.; PALINI, A. (Eds.). Tecnologias alternativas para o controle de pragas e doenças. Viçosa: EPAMIG, 378p. 2006. 